\title{
Exames diagnósticos de síncopes em portadores de marca-passo cardíaco
}

\section{Syncope diagnostic exams in cardiac pacemaker patients}

Gisele Schinaider Cunha ${ }^{1}$. Eduardo Arrais Rocha ${ }^{1}$. Francisca Tatiana Moreira Pereira ${ }^{1}$. Marcelo de Paula Martins Monteiro ${ }^{1}$. Aline Bezerra Tavares ${ }^{2}$. Antônio Brazil Viana Júnior ${ }^{1}$. Ana Rosa Pinto Quidute ${ }^{3}$.

1 Hospital Universitário Walter Cantídio (HUWC), Fortaleza, Ceará, Brasil. 2 Hospital Dr. Carlos Albert Studart Gomes, Fortaleza, Ceará, Brasil. 3 Núcleo de Pesquisa e Desenvolvimento de Fármacos, Universidade Federal do Ceará (UFC), Fortaleza, Ceará, Brasil.

\section{RESUMO}

Introdução: As síncopes podem representar elevado risco de morbimortalidade em determinadas populações. Objetivo: Investigar os resultados dos métodos complementares utilizados no diagnóstico das síncopes em portadores de marca-passos (MP). Metodologia: Foram recrutados participantes do ambulatório de Arritmias e Marca-passo do Hospital Universitário Walter Cantídio, sendo incluídos portadores de MP uni ou bicameral implantados nos últimos 10 anos por doença do nó sinusal ou bloqueio atrioventricular, com capacidade analítica dos eletrogramas endocavitários (EGM), sendo avaliados clinicamente por questionários específicos e análise dos EGM. Demais exames foram solicitados de acordo com a suspeita clínica, como o Holter 24h, Tilt teste, Teste de Hipersensibilidade do Seio Carotídeo ou Estudo Eletrofisiológico. Resultados: O Tilt teste diagnosticou síncopes neuromediadas em quase 1/3 dos casos. A análise computadorizada dos EGM apresentou elevada capacidade diagnóstica, tendo determinado a etiologia arrítmica ou por falhas do MP em 27,6\% dos casos. O Holter 24hs auxiliou na investigação em 18,7\% dos casos. Conclusão: Os testes do marca-passo com as análises de seus eletrogramas armazenados e o Tilt teste foram os melhores métodos para o diagnóstico etiológico das síncopes em portadores de MP cardíaco.

Palavras-chave: Síncope. Marca-passo cardíaco artificial. Técnicas e procedimentos diagnósticos.

\section{ABSTRACT}

Introduction: Syncopes may represent a high risk of morbidity and mortality in certain populations. Objective: To investigate the results of complementary methods used in the diagnosis of syncope in patients with pacemakers (PM). Methods: Participants from the Arrhythmias and Pacemaker Outpatient Unit of the University Hospital Walter Cantídio were recruited. Patients with uni or bicameral PM implanted in the last 10 years due to sinus node or atrioventricular block, with analytical capacity of endocavitary electrograms (EGM) were included and assessed clinically by specific questionnaires and EGM analysis. Other tests were ordered according to clinical suspicion, such as the $24 \mathrm{~h}$ Holter, Tilt test, Carotid sinus hypersensitivity test or an electrophysiology study. Results: The Tilt test diagnosed vasovagal syncopes in almost 1/3 of the cases. The computerized analysis of the EGM presented a high diagnostic capacity, having determined the arrhythmic etiology or PM failures in 27.6\% of the cases. Holter $24 \mathrm{hs}$ assisted in the investigation in $18.7 \%$ of the cases. Conclusion: Pacemaker tests with their stored electrograms and Tilt test were the best methods for etiological diagnosis of syncope in patients with cardiac pacemaker.

Keywords: Syncope. Pacemaker, artificial. Diagnostic techniques and procedures.

Autor correspondente: Gisele Schinaider da Cunha, Rua Capitão Francisco Pedro, 1290, Rodolfo Teófilo, Fortaleza, Ceará. CEP: 60430-372. Telefone: +55 85 99176-6924. E-mail: gisele.schinaider@gmail.com

Conflito de interesses: Não há qualquer conflito de interesses por parte de qualquer um dos autores.

Recebido em: 08 Nov 2018; Revisado em: 13 Out 2019; Aceito em: 07 Dez 2019. 


\section{INTRODUÇÃO}

Síncopes são caracterizadas pela perda transitória de consciência decorrente de hipofluxo cerebral, com perda do tônus postural, sendo súbitas e autolimitadas, de curta duração e que não geram danos neurológicos permanentes. ${ }^{1-2}$

Elas representam um desafio diagnóstico, especialmente nos portadores de marca-passo (MP), nos quais demandam investigação etiológica imediata, por ser um grupo com maior morbimortalidade, geralmente apresentando diversas comorbidades associadas. ${ }^{3-4}$

A determinação da causa pode ser complexa devido à possibilidade de ser multifatorial, e até $30 \%$ permanecem inexplicadas, mesmo após extensa investigação. ${ }^{5} \mathrm{O}$ mau funcionamento do marca-passo ou dos eletrodos pode ser causa das síncopes nesta população, bem como outras etiologias como taquicardia ventricular.,

Diversos métodos complementares são utilizados na investigação da causa da síncope, sejam eles invasivos, como o Estudo Eletrofisiológico (EEF), ou não invasivos, como o Eletrocardiograma, o Holter, o Tilt Test, mas em geral apresentam baixa sensibilidade. Seu uso indevido pode aumentar os custos, o tempo de investigação e causar confusão diagnóstica. Os MP cardíacos modernos apresentam funções diagnósticas cada vez mais sofisticadas, permitindo a análise dos registros eletrocardiográficos endocavitários (EGM).

O presente estudo teve como objetivo investigar os resultados dos métodos complementares utilizados no diagnóstico das síncopes em portadores de marca-passos cardíacos.

\section{MATERIAL E MÉTODOS}

\section{População e desenho do estudo}

Trata-se de estudo de coorte prospectivo, aprovado pelo comitê de ética do Hospital Universitário Walter Cantídio Universidade Federal do Ceará, em que todos os participantes e/ou seus representantes legais assinaram os Termos de Consentimento Livre e Esclarecido (TCLE), de acordo com a resolução 466/2012.

Foram recrutados participantes do ambulatório de Arritmias e Marca-passo da instituição, sendo incluídos portadores de marca-passo (MP) uni ou bicameral, implantados nos últimos 10 anos por doença do nó sinusal ou bloqueio atrioventricular, com capacidade de análises dos eletrogramas endocavitários (EGM).

Foram excluídos os portadores de Cardiodesfibrilador Implantável (CDI) e de marca-passos multissítio ou biventriculares. Aqueles que tiveram pelo menos um episódio de síncope nos últimos doze meses foram alocados no grupo caso, e aqueles sem histórico de síncopes e portadores de $\mathrm{MP}$, para o grupo controle. $\mathrm{O}$ acompanhamento era realizado trimestralmente no $1^{\mathrm{a}}$ ano ou antes em caso de recorrência do episódio.
Aseleção dos pacientes ocorreu entre maio/2015 e janeiro/2018. O protocolo de investigação incluía uma história detalhada dos episódios por meio de questionários padronizados (Figura 1) realizados por cardiologistas especializados em arritmia, além de exame físico com pesquisa de hipotensão ortostática (HO), avaliação dos MP computadorizada pelos programadores específicos, com análise dos eletrogramas endocavitários (EGM), com testes de inibição por miopotenciais, além da realização do Eletrocardiograma e Ecocardiograma (ECO).

Após avaliação clínica inicial eram solicitados outros exames complementares de acordo com a suspeita clínica, como o Holter 24h, Holter 7 dias, Tilt teste, Teste de Hipersensibilidade do Seio Carotídeo, Estudo Eletrofisiológico ou exames de avaliação neurológica.

Figura 1. Questionário de avaliação dos pacientes.

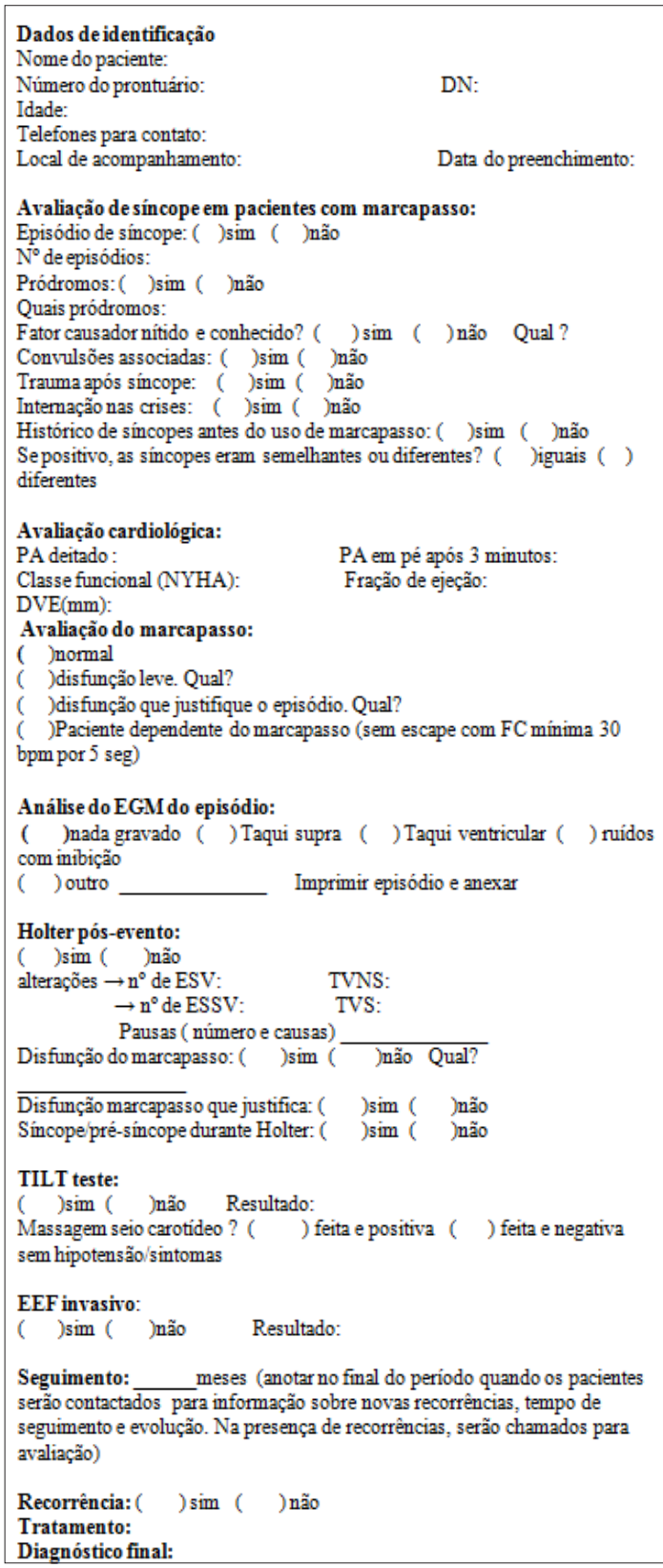




\section{Análise estatística}

As variáveis contínuas foram analisadas pelo método de Mann-Whitney e as categóricas pelo testes $\chi^{2}$ ou Fischer, quando apropriado. Em todos os testes foi adotado o nível de significância de $5 \%(\mathrm{p}<0,05)$.

\section{RESULTADOS}

Ocorreram 100 episódios de síncope em 47 indivíduos, dentre os quais 14 tiveram dois eventos e 11 deles tiveram mais de três. Foram incluídos 95 pacientes portadores de MP no estudo, sendo 48 sem síncopes e 47 com pelo menos um episódio de síncope nos últimos 12 meses. As características clínicas dos 95 pacientes estão apresentadas na Tabela 1. O tempo de seguimento médio foi de 18,5 meses.

Nos pacientes com causas cardíacas, ocorreram três casos de fibrilação atrial (FA) com alta resposta, quatro casos de taquicardia ventricular sustentada (TVS) e uma fibrilação ventricular (FV) após síndrome coronariana aguda. Três pacientes apresentaram crises convulsivas e um teve síncope no contexto de um acidente vascular encefálico. No grupo de causas diversas houve duas perdas de consciência em episódios de hipoglicemia.

Não ocorreram diferenças entre os grupos caso e controle com relação ao gênero $(p=0,61)$, idade $(p=0,40)$, fração de ejeção $(p=0,77)$ e diâmetro do ventrículo esquerdo $(p=0,266)$.

A análise computadorizada dos MP foi capaz de determinar a etiologia das síncopes em $27,6 \%$ dos casos. Quatro ocorreram por falhas de comando ou sense (três por aumento de limiar e um por fratura de eletrodo), e em oito casos foram registradas arritmias, sendo quatro casos de taquicardia ventricular sustentada, três de fibrilação atrial de alta resposta, uma fibrilação ventricular após síndrome coronarianaaguda (paciente desfibrilado imediatamente) e uma síncope durante um infarto com supradesnível do segmento $\mathrm{ST}$, sem registro de arritmias concomitantes.

O Holter de $24 \mathrm{~h}$ se mostrou como bom auxílio na investigação em 18,7\% dos casos, tendo, entretanto, poder diagnóstico em apenas 3\%. O Tilt teste foi diagnóstico em 28,5\% dos casos, a massagem do seio carotídeo em um caso e o Estudo Eletrofisiológico Invasivo, realizado em nove pacientes, teve resultados inconclusivos em todos.

Tabela 1. Características dos pacientes do estudo.

\begin{tabular}{lllc}
\hline \multicolumn{1}{c}{ Variável } & Grupo com síncope & Grupo sem síncope & Nível p \\
\hline Gênero (\% masculino) & $51 \%$ & $54,1 \%$ & 0,61 \\
Idade média (anos) & $78,1 \pm 12,1$ & $76,9 \pm 11,5$ & 0,40 \\
$\geq 3$ comorbidades & $59,5 \%$ & $35,4 \%$ & $\mathbf{0 , 0 1}$ \\
& I: $38,2 \%$ & I: $89,5 \%$ & $<\mathbf{0 , 0 0 1}$ \\
NYHA* & II: $48,9 \%$ & II: $8,3 \%$ & $<\mathbf{0 , 0 0 1}$ \\
& III: $12,7 \%$ & III: $2 \%$ & $<\mathbf{0 , 0 0 1}$ \\
Dependente do MP & $34 \%$ & $8,3 \%$ & $\mathbf{0 , 0 2}$ \\
Cardiopatia estrutural & $59,5 \%$ & $41,6 \%$ & $\mathbf{0 , 0 3 6}$ \\
Fração de Ejeção (\%) & $57,0 \pm 12,1$ & $57,7 \pm 7,1$ & 0,77 \\
DDVE (mm) & $51,3 \pm 5,2$ & $50,2 \pm 6,2$ & 0,26 \\
\hline
\end{tabular}

NYHA: Classe Funcional de New York Heart Association; *: não houve pacientes NYHA IV no estudo; FEVE: Fração de Ejeção de Ventrículo Esquerdo; DDVE: diâmetro diastólico do Ventrículo Esquerdo. Comorbidades: presença de um 3 ou mais dos itens, como síndrome de fragilidade, AVC prévio, diabetes, insuficiência cardíaca, DPOC, vasculopatia. Nível p de significância estatística $p<0,05$.

\section{DISCUSSÃO}

Dentre os exames utilizados na investigação da etiologia da síncope, o Tilt teste se destacou, sendo capaz de dar diagnóstico de síncope neuromediada em quase $1 / 3$ dos casos. Ele pode avaliar a maior chance de recorrência das síncopes reflexas, ${ }^{3}$ com elevada capacidade diagnóstica nos portadores de MP que evoluem com síncope e apresentam sintomas posturais. ${ }^{4}$

Na realidade, os resultados do Tilt teste devem ser interpretados como uma maior susceptibilidade à hipotensão relacionada ao stress postural, capaz de identificar uma tendência individual à ocorrência de síncopes por este mecanismo, e não simplesmente como teste capaz de elucidar o diagnóstico, ressaltando o caráter multifatorial da condição ${ }^{1,3}$ e a ausência de um padrão-ouro comparativo para se afirmar ser o resultado verdadeiro positivo.

Portanto, a informação fornecida pelo paciente com relação à semelhança dos episódios é muito importante para confirmação diagnóstica, e não apenas a informação de positividade, com chances de resultado falso-positivo.

A elevada capacidade diagnóstica das análises dos EGM dos MP vai ao encontro da evolução das diversas técnicas de 
monitorização prolongada que revolucionaram o diagnóstico de síncope e reduziram as taxas de síncopes inexplicadas para valores abaixo de $10-20 \%$. Os MP mais recentes têm aprimorado a morfologia dos seus traçados, permitindo uma análise eletrocardiográfica mais adequada. ${ }^{6}$

O exame de Holter pode eventualmente detectar falhas de comando intermitente ou inibições inadequadas que não tenham sido verificadas durante os testes de programação, sendo um exame de baixo custo que deve ser sempre solicitado, apesar de sua baixa sensibilidade diagnóstica. Alguns pacientes tiveram seus diagnósticos após realização

\section{REFERÊNCIAS}

1. Brignole M, Moya A, Lange FJ, Deharo JC, Elliott PM.; Fanciulli A, et al. ESC guidelines for the diagnosis and management of syncope. Eur Heart J. 2018;39(21):1883-1948.

2. Shen WK, Sheldon RS, Benditt DG, Cohen MI, Forman DE, Goldberger ZD, et al. ACC/AHA/HRS Guideline for the evaluation and management of patients with syncope. J Am Coll Cardiol. 2017;70(5):39-110.

3. Sutton R. Syncope in patients with pacemakers. Arrhythm Electrophysiol Rev. 2015;4(3):189-92. de exames repetidos de Holter ou com o exame de Holter de sete dias. Em nossa casuística, optamos por realizar estudo eletrofisiológico invasivo (EEF) em nove pacientes em virtude de registros endocavitários não adequados e suspeita clínica elevada de síncope por taquiarritmia.

\section{CONCLUSÕES}

Os testes do marca-passo com as análises de seus eletrogramas armazenados e o Tilt teste foram os melhores métodos para diagnosticar as causas de síncopes nos portadores de marca-passos cardíacos.

4. Haarmark C, Kanters JK, Mehlsen J. Tilt-table testing of patients with pacemaker and recurrent syncope. Indian Pacing Electrophysiol J. 2015;15(4):193-8.

5. Bhargava K. Tilt test in paced patients: Is it worth the effort?. Indian Pacing Electrophysiol J. 2015;15(4):189-92.

6. Ofman P, Rahilly-Tierney C, Djousse L, Peralta A, Hoffmeister $\mathrm{P}$, Gaziano MJ, et al. Pacing system malfunction is a rare cause of hospital admission for syncope in patients with a permanent pacemaker. Pacing Clin Electrophysiol. 2012;36(1):109-12.

\section{Como citar:}

Cunha GS, Rocha EA, Pereira FT, Monteiro MP, Tavares AB, Viana AB Júnior, et al. Exames diagnósticos de síncopes em portadores de marca-passo cardíaco. Rev Med UFC. 2020 jul-set;60(3):11-14. 